\title{
General $L_{p}$-mixed chord integrals of star bodies
}

\section{Zhaofeng Li* and Weidong Wang}

"Correspondence: kelly0128@163.com

Department of Mathematics, China Three Gorges University, Yichang, 443002, China (c) $2016 \mathrm{Li}$ and Wang. This article is distributed under the terms of the Creative Commons Attribution 4.0 International License (http://creativecommons.org/licenses/by/4.0/), which permits unrestricted use, distribution, and reproduction in any medium, provided you give appropriate credit to the original author(s) and the source, provide a link to the Creative Commons license, and indicate if changes were made.

\begin{abstract}
The notion of general mixed chord integrals of star bodies was introduced by Feng and Wang. In this paper, we extend the concept of the general mixed chord integrals to general $L_{p}$-mixed chord integrals of star bodies. Based on this new notion, we study their extremum values and obtain an Aleksandrov-Frenchel type and a cyclic inequality for general $L_{p}$-mixed chord integrals of star bodies, respectively. Further, as applications, we establish two Brunn-Minkowski type inequalities for $L_{p}$-radial bodies. Finally, we get an interesting identical equality on combining $L_{p}$-radial bodies.
\end{abstract}

MSC: 52A20; 52A40; 52A39

Keywords: $s t a r$ body; mixed chord integrals; general $L_{p}$-mixed chord integrals; $L_{p}$-radial body

\section{Introduction and main results}

The setting for this paper is $n$-dimensional Euclidean spaces $\mathbb{R}^{n}$. Let $\mathcal{K}^{n}$ denote the set of convex bodies (compact, convex subsets with nonempty interiors) in $\mathbb{R}^{n}$. $\mathcal{K}_{0}^{n}$ denotes the set of convex bodies containing the origin in their interiors. The $n$-dimensional volume of a body $K$ is written as $V(K)$. The unit sphere in $\mathbb{R}^{n}$ is denoted $\mathcal{S}^{n-1}$ and $B$ denotes the standard unit ball in $\mathbb{R}^{n}$.

If $K$ is a compact star shaped (about the origin) set in $\mathbb{R}^{n}$, then its radial function, $\rho_{K}=$ $\rho(K, \cdot): \mathbb{R}^{n} \backslash\{0\} \rightarrow[0, \infty)$, is defined by (see $\left.[1,2]\right)$

$$
\rho(K, u)=\max \{\lambda \geq 0, \lambda u \in K\}, \quad u \in \mathcal{S}^{n-1} .
$$

If $\rho_{K}$ is positive and continuous, then $K$ is called a star body (about the origin) and $\mathcal{S}^{n}$ denotes the set of star bodies in $\mathbb{R}^{n}$. We will use $\mathcal{S}_{0}^{n}$ to denote the subset of $\mathcal{S}^{n}$ containing the origin in their interiors. Two star bodies $K$ and $L$ are said to be dilated of one another if $\rho_{K}(u) / \rho_{L}(u)$ is independent of $u \in \mathcal{S}^{n-1}$.

The notion of mixed chord integrals of star bodies was defined by Lu (see [3]). Feng and Wang (see [4]) generalized the definition of mixed chord integrals to the general mixed chord integrals of star bodies: For $K_{1}, \ldots, K_{n} \in \mathcal{S}_{0}^{n}$ and $\tau \in(-1,1)$, the general mixed chord integral $C^{(\tau)}\left(K_{1}, \ldots, K_{n}\right)$ is defined by

$$
C^{(\tau)}\left(K_{1}, \ldots, K_{n}\right)=\frac{1}{n} \int_{\mathcal{S}^{n-1}} c^{(\tau)}\left(K_{1}, u\right) \cdots c^{(\tau)}\left(K_{n}, u\right) d u,
$$

(n) 
where $c^{(\tau)}(K, \cdot)=f_{1}(\tau) \rho(K, \cdot)+f_{2}(\tau) \rho(-K, \cdot)$ and the functions $f_{1}(\tau)$ and $f_{2}(\tau)$ are defined as follows:

$$
f_{1}(\tau)=\frac{(1+\tau)^{2}}{2\left(1+\tau^{2}\right)}, \quad f_{2}(\tau)=\frac{(1-\tau)^{2}}{2\left(1+\tau^{2}\right)}
$$

The general mixed chord integral is a map $\underbrace{\mathcal{S}_{o}^{n} \times \cdots \times \mathcal{S}_{o}^{n}}_{n} \rightarrow \mathbb{R}$. It is positive, continuous, multilinear with respect to radial Minkowski linear combinations, positively homogeneous and monotone under set inclusion. Star bodies $K_{1}, \ldots, K_{n}$ are said to have a similar chord if there exist constants $\lambda_{1}, \ldots, \lambda_{n}>0$ such that $\lambda_{1} c^{(\tau)}\left(K_{1}, u\right)=\cdots=\lambda_{n} c^{(\tau)}\left(K_{n}, u\right)$ for all $u \in S^{n-1}$. Feng and Wang (see [4]) established the following inequalities.

Theorem 1.A If $\tau \in(-1,1)$ and $K_{1}, \ldots, K_{n} \in \mathcal{S}_{o}^{n}$, then

$$
C^{(\tau)}\left(K_{1}, \ldots, K_{n}\right)^{n} \leq V\left(K_{1}\right) \cdots V\left(K_{n}\right)
$$

with equality if and only if $K_{1}, \ldots, K_{n}$ are dilates of each other centered.

Theorem 1.B If $\tau \in(-1,1)$ and $K_{1}, \ldots, K_{n} \in \mathcal{S}_{o}^{n}$, then for $1<m \leq n$,

$$
C^{(\tau)}\left(K_{1}, \ldots, K_{n}\right)^{m} \leq \prod_{i=1}^{m} C^{(\tau)}\left(K_{1}, \ldots, K_{n-m}, K_{n-i+1}, K_{n-i+1}, \ldots, K_{n-i+1}\right),
$$

with equality if and only if $K_{n-m+1}, \ldots, K_{n}$ are all of similar general chord.

Theorem 1.C If $\tau \in(-1,1)$ and $K, L \in \mathcal{S}_{o}^{n}$, then for $i<j<k$,

$$
C_{i}^{(\tau)}(K, L)^{k-j} C_{k}^{(\tau)}(K, L)^{j-i} \geq C_{j}^{(\tau)}(K, L)^{k-i}
$$

with equality if and only if $K$ and $L$ have a similar general chord.

Here $C_{i}^{(\tau)}(K, L)=C_{i}^{(\tau)}(K, n-i ; L, i)$ in which $K$ appears $n-i$ times and $L$ appears $i$ times. Motivated by Lu and Feng and Wang (see [3, 4]), in this paper, we define the general $L_{p}$-mixed chord integrals as follows: For $K_{1}, \ldots, K_{n} \in \mathcal{S}_{0}^{n}, p>0$, and $\tau \in[-1,1]$, the general $L_{p}$-mixed chord integral $C_{p}^{(\tau)}\left(K_{1}, \ldots, K_{n}\right)$ of $K_{1}, \ldots, K_{n}$ is defined by

$$
C_{p}^{(\tau)}\left(K_{1}, \ldots, K_{n}\right)=\frac{1}{n} \int_{\mathcal{S}^{n-1}} c_{p}^{(\tau)}\left(K_{1}, u\right) \cdots c_{p}^{(\tau)}\left(K_{n}, u\right) d u
$$

Here $c_{p}^{(\tau)}(K, \cdot)$ is defined by

$$
c_{p}^{(\tau)}(K, u)=\left(f_{1}(\tau) \rho^{p}(K, u)+f_{2}(\tau) \rho^{p}(-K, u)\right)^{\frac{1}{p}}
$$

for any $u \in \mathcal{S}^{n-1}$, and $f_{1}(\tau)$ and $f_{2}(\tau)$ are chosen as (see [5])

$$
f_{1}(\tau)=\frac{(1+\tau)^{p}}{(1+\tau)^{p}+(1-\tau)^{p}}, \quad f_{2}(\tau)=\frac{(1-\tau)^{p}}{(1+\tau)^{p}+(1-\tau)^{p}} .
$$


Obviously, $f_{1}(\tau)$ and $f_{2}(\tau)$ satisfy

$$
\begin{aligned}
& f_{1}(\tau)+f_{2}(\tau)=1 ; \\
& f_{1}(-\tau)=f_{2}(\tau), \quad f_{2}(-\tau)=f_{1}(\tau) .
\end{aligned}
$$

The general $L_{p}$-mixed chord integral is also a map $\underbrace{\mathcal{S}_{o}^{n} \times \cdots \times \mathcal{S}_{o}^{n}}_{n} \rightarrow \mathbb{R}$ and it is positive, continuous, multilinear with respect to $L_{p}$-radial Minkowski linear combinations, positively homogeneous and monotone under set inclusion. If there exist constants $\lambda_{1}, \ldots, \lambda_{n}>$ 0 such that $\lambda_{1} c_{p}^{(\tau)}\left(K_{1}, u\right)=\cdots=\lambda_{n} c_{p}^{(\tau)}\left(K_{n}, u\right)$ for all $u \in \mathcal{S}^{n-1}$, star bodies $K_{1}, \ldots, K_{n}$ are said to have a similar general $L_{p}$-chord.

Let $\underbrace{K_{1}=\cdots=K_{n-i}}_{n-i}=K$ and $\underbrace{K_{n-i+1}=\cdots=K_{n}}_{i}=L(i=0,1, \ldots, n)$ in (1.1), we denote $C_{p, i}^{(\tau)}(K, L)$ in which $K$ appears $n-i$ times and $L$ appears $i$ times. From this, if $i$ is any real, we define the following.

For $K, L \in \mathcal{S}_{o}^{n}, p>0, \tau \in[-1,1]$, and $i$ any real, the $i$ th general $L_{p}$-mixed chord integral, $C_{p, i}^{(\tau)}(K, L)$, of $K$ and $L$ is given by

$$
C_{p, i}^{(\tau)}(K, L)=\frac{1}{n} \int_{\mathcal{S}^{n-1}} c_{p}^{(\tau)}(K, u)^{n-i} c_{p}^{(\tau)}(L, u)^{i} d u
$$

Further, if $L=B$ in (1.3), we write $C_{p, i}^{(\tau)}(K, B)$ as $C_{p, i}^{(\tau)}(K)$ and notice that $c_{p}^{(\tau)}(B, u)=1$, we have

$$
C_{p, i}^{(\tau)}(K)=\frac{1}{n} \int_{\mathcal{S}^{n-1}} c_{p}^{(\tau)}(K, u)^{n-i} d u
$$

where $C_{p, i}^{(\tau)}(K)$ is called the $i$ th general $L_{p}$-mixed chord integral of $K$. If $i=0$, we write $C_{p, 0}^{(\tau)}(K)=C_{p}^{(\tau)}(K)$, which we call the general $L_{p}$-chord integral of $K$, that is,

$$
C_{p}^{(\tau)}(K)=\frac{1}{n} \int_{\mathcal{S}^{n-1}} c_{p}^{(\tau)}(K, u)^{n} d u .
$$

The general $L_{p}$-mixed chord integrals of $K$ belong to a new and rapidly evolving asymmetric $L_{p}$ Brunn-Minkowski theory that has its origins in the work of Ludwig et al. (see [5-11]). For the further research of asymmetric $L_{p}$ Brunn-Minkowski theory, also see [1228].

In this paper, motivated by Feng and Wang (see [4]), we deduce several inequalities for the general $L_{p}$-mixed chord integrals.

Theorem 1.1 If $\tau \in[-1,1]$ and $K_{1}, \ldots, K_{n} \in \mathcal{S}_{o}^{n}$, then for $p<n$,

$$
C_{p}^{(\tau)}\left(K_{1}, \ldots, K_{n}\right)^{n} \leq V\left(K_{1}\right) \cdots V\left(K_{n}\right)
$$

for $p>n$,

$$
C_{p}^{(\tau)}\left(K_{1}, \ldots, K_{n}\right)^{n} \geq V\left(K_{1}\right) \cdots V\left(K_{n}\right)
$$

with equality if and only if $K_{1}, \ldots, K_{n}$ are dilates of each other. 
Theorem 1.2 If $K \in \mathcal{S}_{o}^{n}$ and $\tau \in[-1,1], p>0$, then for $i<n-p$,

$$
C_{p, i}^{(0)}(K) \leq C_{p, i}^{(\tau)}(K) \leq C_{p, i}^{( \pm 1)}(K)
$$

for $n-p<i<n$ or $i>n$,

$$
C_{p, i}^{(0)}(K) \leq C_{p, i}^{(\tau)}(K) \leq C_{p, i}^{( \pm 1)}(K)
$$

If $K$ is not origin-symmetric, equality holds in the left inequality if and only if $\tau=0$ and equality holds in the right inequality if and only if $\tau= \pm 1$. When $i=n,(1.7)$ and (1.8) are identical.

Theorem 1.3 If $K_{1}, \ldots, K_{n} \in \mathcal{S}_{o}^{n}$ and $\tau \in[-1,1], p>0$, then for $1<m \leq n$,

$$
C_{p}^{(\tau)}\left(K_{1}, \ldots, K_{n}\right)^{m} \leq \prod_{i=1}^{m} C_{p}^{(\tau)}\left(K_{1}, \ldots, K_{n-m}, K_{n-i+1}, K_{n-i+1}, \ldots, K_{n-i+1}\right),
$$

with equality if and only if $K_{n-m+1}, \ldots, K_{n}$ all have a similar general $L_{p}$-chord.

Theorem 1.4 If $K, L \in \mathcal{S}_{o}^{n}$ and $\tau \in[-1,1], p>0$, then, for $i<j<k$,

$$
C_{p, j}^{(\tau)}(K, L)^{k-i} \leq C_{p, i}^{(\tau)}(K, L)^{k-j} C_{p, k}^{(\tau)}(K, L)^{j-i}
$$

with equality if and only if $K$ and $L$ have a similar general $L_{p}$-chord.

Further, as applications, for the $L_{p}$-radial combination, we give two Brunn-Minkowski type inequalities for the general $L_{p}$-mixed chord integrals as follows.

Theorem 1.5 If $K, L \in \mathcal{S}_{o}^{n}$ and $\tau \in[-1,1], p>0, \lambda, \mu \geq 0$ (not both zero), then for $i \leq n-p$,

$$
C_{p, i}^{(\tau)}\left(\lambda \circ K \tilde{+}_{p} \mu \circ L\right)^{\frac{p}{n-i}} \leq \lambda C_{p, i}^{(\tau)}(K)^{\frac{p}{n-i}}+\mu C_{p, i}^{(\tau)}(L)^{\frac{p}{n-i}} ;
$$

for $n-p<i<n$ or $i>n$,

$$
C_{p, i}^{(\tau)}\left(\lambda \circ K \tilde{+}_{p} \mu \circ L\right)^{\frac{p}{n-i}} \geq \lambda C_{p, i}^{(\tau)}(K)^{\frac{p}{n-i}}+\mu C_{p, i}^{(\tau)}(L)^{\frac{p}{n-i}},
$$

with equality in each inequality if and only if $K$ and $L$ have a similar general $L_{p}$-chord. Here and in the following theorem $\lambda \circ K \tilde{+}_{p} \mu \circ L$ denotes the $L_{p}$-radial Minkowski combination of $K$ and $L$.

Theorem 1.6 If $K, L \in \mathcal{S}_{o}^{n}$ and $\tau \in[-1,1], p>0$, then for $i \leq n-p \leq j \leq n$,

$$
\left(\frac{C_{p, i}^{(\tau)}\left(K \tilde{+}_{p} L\right)}{C_{p, j}^{(\tau)}\left(K \tilde{+}_{p} L\right)}\right)^{\frac{p}{j-i}} \leq\left(\frac{C_{p, i}^{(\tau)}(K)}{C_{p, j}^{(\tau)}(K)}\right)^{\frac{p}{j-i}}+\left(\frac{C_{p, i}^{(\tau)}(L)}{C_{p, j}^{(\tau)}(L)}\right)^{\frac{p}{j-i}}
$$

for $j \geq n \geq i \geq n-p$, inequality (1.13) is reversed, with equality in each inequality if and only if $K$ and $L$ have a similar general $L_{p}$-chord. 
Finally, we get an interesting identical equality for the $L_{p}$-radial bodies.

Theorem 1.7 If $K \in \mathcal{S}_{o}^{n}$ and $p>0$, then for $\forall \tau \in[-1,1]$, we have

$$
C_{p, i}^{(\tau)}\left(\bar{\triangle}_{p} K\right)=C_{p, i}(K)
$$

The proofs of Theorems 1.1-1.7 are given in Section 3 of this paper. In the following section, we summarize some associated properties of the general $L_{p}$-mixed chord integrals needed in the proof of main results.

\section{Preliminaries}

The dual quermassintegral was defined by Lutwak (see [29]) as follows. For $K \in \mathcal{S}_{o}^{n}$ and any real $i$, the dual quermassintegral, $\widetilde{W}_{i}(K)$, of $K$ is defined by

$$
\widetilde{W}_{i}(K)=\frac{1}{n} \int_{\mathcal{S}^{n-1}} \rho(K, u)^{n-i} d u
$$

obviously, (2.1) implies the volume $V(K)$ of $K$ is

$$
V(K)=\widetilde{W}_{0}(K)=\frac{1}{n} \int_{\mathcal{S}^{n-1}} \rho(K, u)^{n} d u
$$

If $E$ is a nonempty set in $\mathbb{R}^{n}$, then the polar set of $E$, $E^{*}$, is defined by (see [1])

$$
E^{*}=\left\{x \in \mathbb{R}^{n}: x \cdot y \leq 1 \text {, any } y \in E\right\} .
$$

For $K \in \mathcal{S}_{o s}^{n}$, an extension of the Blaschke-Santaló inequality takes the following form (see [30]).

Theorem 2.A If $K \in \mathcal{S}_{o s}^{n}$, then

$$
V(K) V\left(K^{*}\right) \leq \omega_{n}^{2}
$$

with equality if and only if $K$ is an ellipsoid. Here $\omega_{n}=V(B)$.

For $K, L \in \mathcal{S}_{o}^{n}, p>0$, and $\lambda, \mu \geq 0$ (not both zero), the Firey $L_{p}$-radial combination, $\lambda \circ$ $K \tilde{+}_{p} \mu \circ L$, of $K$ and $L$ is defined by (see [6, 31])

$$
\rho\left(\lambda \circ K \tilde{+}_{p} \mu \circ L, \cdot\right)^{p}=\lambda \rho(K, \cdot)^{p}+\mu \rho(L, \cdot)^{p},
$$

here $\lambda \circ K$ denotes the $L_{p}$-radial scalar multiplication and $\lambda \circ K=\lambda^{\frac{1}{p}} K$.

In (2.4), let $\lambda=\mu=\frac{1}{2}$ and let $L=-K$, we have the definition of $L_{p}$-radial body $\bar{\triangle}_{p} K$ as follows (see [31]):

$$
\bar{\Delta}_{p} K=\frac{1}{2} \circ K \tilde{+}_{p} \frac{1}{2} \circ(-K) .
$$




\section{Proofs of main results}

In this section, we prove Theorems 1.1-1.7. The proofs of Theorems 1.1 and 1.2 require the following lemma.

Lemma 3.1 If $K \in \mathcal{S}_{o}^{n}$ and $\tau \in[-1,1], p>0$, then for $i<n-p$,

$$
C_{p, i}^{(\tau)}(K) \leq \widetilde{W}_{i}(K)
$$

for $n-p<i<n$ or $i>n$,

$$
C_{p, i}^{(\tau)}(K) \leq \widetilde{W}_{i}(K)
$$

with equality if and only if $K$ is centered. When $i=n,(3.1)$ and (3.2) are identical.

Proof From definitions (1.1) and (1.4), and using Minkowski's inequality (see [32]), for $i<$ $n-p$, we have

$$
\begin{aligned}
C_{p, i}^{(\tau)}(K)^{\frac{p}{n-i}}= & {\left[\frac{1}{n} \int_{\mathcal{S}^{n-1}} c_{p}^{(\tau)}(K, u)^{n-i} d u\right]^{\frac{p}{n-i}} } \\
= & {\left[\frac{1}{n} \int_{\mathcal{S}^{n-1}}\left(f_{1}(\tau) \rho^{p}(K, u)+f_{2}(\tau) \rho^{p}(-K, u)\right)^{\frac{n-i}{p}} d u\right]^{\frac{p}{n-i}} } \\
\leq & {\left[\frac{1}{n} \int_{\mathcal{S}^{n-1}}\left(f_{1}(\tau) \rho^{p}(K, u)\right)^{\frac{n-i}{p}} d u\right]^{\frac{p}{n-i}} } \\
& +\left[\frac{1}{n} \int_{\mathcal{S}^{n-1}}\left(f_{2}(\tau) \rho^{p}(-K, u)\right)^{\frac{n-i}{p}} d u\right]^{\frac{p}{n-i}} \\
= & {\left[\frac{1}{n} \int_{\mathcal{S}^{n-1}}\left(\rho^{p}(K, u)\right)^{\frac{n-i}{p}} d u\right]^{\frac{p}{n-i}} } \\
= & {\left[\frac{1}{n} \int_{\mathcal{S}^{n-1}} \rho(K, u)^{n-i} d u\right]^{\frac{p}{n-i}} }
\end{aligned}
$$

i.e.

$$
C_{p, i}^{(\tau)}(K) \leq \frac{1}{n} \int_{\mathcal{S}^{n-1}} \rho(K, u)^{n-i} d u=\widetilde{W}_{i}(K) .
$$

Similarly, for $n-p<i<n$ or $i<n$, by Minkowski's inequality we know (3.2) holds. From the equality condition of Minkowski's inequality, we see that equalities hold in (3.1) and (3.2) if and only if $K$ and $-K$ are dilates of one another, that is, $K$ is centered.

In Lemma 3.1, let $i=0$ and notice (2.2), we have the following corollary immediately.

Corollary 3.1 If $K \in \mathcal{S}_{o}^{n}$ and $\tau \in[-1,1], p>0$, then for $p<n$,

$$
C_{p}^{(\tau)}(K) \leq V(K)
$$


for $p>n$,

$$
C_{p}^{(\tau)}(K) \geq V(K)
$$

with equality if and only if $K$ is centered.

Theorem 2.A leads to the following corollary.

Corollary 3.2 If $K \in \mathcal{S}_{o s}^{n}$ and $\tau \in[-1,1], p>0$, then for $p<n$ and $n \geq 1$,

$$
C_{p}^{(\tau)}(K) C_{p}^{(\tau)}\left(K^{*}\right) \leq \omega_{n}^{2}
$$

with equality in each inequality if and only if $K$ is an ellipsoid centered at the origin.

Proof of Theorem 1.1 Using Hölder's inequality (see [32]), we have for $p<n$ and $n \geq 1$,

$$
\begin{aligned}
C_{p}^{(\tau)}\left(K_{1}, \ldots, K_{n}\right) & =\left[\frac{1}{n} \int_{\mathcal{S}^{n-1}} c_{p}^{(\tau)}\left(K_{1}, u\right) \cdots c_{p}^{(\tau)}\left(K_{n}, u\right) d u\right]^{n \cdot \frac{1}{n}} \\
& \leq\left[\frac{1}{n} \int_{\mathcal{S}^{n-1}}\left(c_{p}^{(\tau)}\left(K_{1}, u\right)\right)^{n} d u\right]^{\frac{1}{n}} \times \cdots \times\left[\frac{1}{n} \int_{\mathcal{S}^{n-1}}\left(c_{p}^{(\tau)}\left(K_{n}, u\right)\right)^{n} d u\right]^{\frac{1}{n}} \\
& =C_{p}^{(\tau)}\left(K_{1}\right)^{\frac{1}{n}} \cdots C_{p}^{(\tau)}\left(K_{n}\right)^{\frac{1}{n}} \\
& \leq V\left(K_{1}\right)^{\frac{1}{n}} \cdots V\left(K_{n}\right)^{\frac{1}{n}}
\end{aligned}
$$

i.e. $C_{p}^{(\tau)}\left(K_{1}, \ldots, K_{n}\right)^{n} \leq V\left(K_{1}\right) \cdots V\left(K_{n}\right)$.

Similarly, we can obtain $C_{p}^{(\tau)}\left(K_{1}, \ldots, K_{n}\right)^{n} \geq V\left(K_{1}\right) \cdots V\left(K_{n}\right)$ when $p>n$. The equality conditions of (3.3) and (3.4) tell us that equalities hold in Theorem 1.1 if and only if $K_{1}, \ldots, K_{n}$ are dilates of each other.

Proof of Theorem 1.2 From the proof of Lemma 3.1, for $i<n-p$, we get

$$
C_{p, i}^{(\tau)}(K) \leq \frac{1}{n} \int_{\mathcal{S}^{n-1}}\left(\rho^{p}(K, u)\right)^{\frac{n-i}{p}} d u \quad \text { or } \quad \frac{1}{n} \int_{\mathcal{S}^{n-1}}\left(\rho^{p}(-K, u)\right)^{\frac{n-i}{p}} d u .
$$

Notice (1.2), $f_{1}(+1)=1, f_{2}(+1)=0$, we have

$$
C_{p, i}^{(+1)}(K)=\frac{1}{n} \int_{\mathcal{S}^{n-1}}\left(\rho^{p}(K, u)\right)^{\frac{n-i}{p}} d u
$$

Similarly, we obtain

$$
C_{p, i}^{(-1)}(K)=\frac{1}{n} \int_{\mathcal{S}^{n-1}}\left(\rho^{p}(-K, u)\right)^{\frac{n-i}{p}} d u
$$

Together with (3.6), it follows that

$$
C_{p, i}^{(\tau)}(K) \leq C_{p, i}^{( \pm 1)}(K)
$$

which is just the right inequality of (1.7). 
According the equality condition of Lemma 3.1, we know that if $\tau \neq \pm 1$, equality holds in the right inequality of (1.7) if and only if $K$ is centered, that means that, if $K$ is not origin-symmetric, equality holds in the right inequality if and only if $\tau= \pm 1$.

Now we prove the left inequality of (1.7). When $\tau=0$, we have $f_{1}(\tau)=f_{2}(\tau)=\frac{1}{2}$, thus, by Minkowski's inequality,

$$
\begin{aligned}
C_{p, i}^{(0)}(K)^{\frac{p}{n-i}}= & {\left[\frac{1}{n} \int_{\mathcal{S}^{n-1}}\left(\frac{1}{2} \rho^{p}(K, u)+\frac{1}{2} \rho^{p}(-K, u)\right)^{\frac{n-i}{p}} d u\right]^{\frac{p}{n-i}} } \\
= & {\left[\frac { 1 } { n } \int _ { \mathcal { S } ^ { n - 1 } } \left(\frac{1}{2} f_{1}(\tau) \rho^{p}(K, u)+\frac{1}{2} f_{2}(\tau) \rho^{p}(-K, u)\right.\right.} \\
& \left.\left.+\frac{1}{2} f_{1}(\tau) \rho^{p}(-K, u)+\frac{1}{2} f_{2}(\tau) \rho^{p}(K, u)\right)^{\frac{n-i}{p}} d u\right]^{\frac{p}{n-i}} \\
\leq & {\left[\frac{1}{n} \int_{\mathcal{S}^{n-1}}\left(\frac{1}{2} f_{1}(\tau) \rho^{p}(K, u)+\frac{1}{2} f_{2}(\tau) \rho^{p}(-K, u)\right)^{\frac{n-i}{p}} d u\right]^{\frac{p}{n-i}} } \\
& +\left[\frac{1}{n} \int_{\mathcal{S}^{n-1}}\left(\frac{1}{2} f_{1}(\tau) \rho^{p}(-K, u)+\frac{1}{2} f_{2}(\tau) \rho^{p}(K, u)\right)^{\frac{n-i}{p}} d u\right]^{\frac{p}{n-i}} \\
= & {\left[\frac{1}{n} \int_{\mathcal{S}^{n-1}}\left(f_{1}(\tau) \rho^{p}(K, u)+f_{2}(\tau) \rho^{p}(-K, u)\right)^{\frac{n-i}{p}} d u\right]^{\frac{p}{n-i}} } \\
= & {\left[\frac{1}{n} \int_{\mathcal{S}^{n-1}} c_{p}^{(\tau)}(K, u)^{n-i} d u\right]^{\frac{p}{n-i}} } \\
= & C_{p, i}^{(\tau)}(K)^{\frac{p}{n-i}}
\end{aligned}
$$

i.e. $C_{p, i}^{(0)}(K) \leq C_{p, i}^{(\tau)}(K)$, which just is the left inequality of (1.7).

The equality condition of Minkowski's inequality tells us that if $\tau \neq 0$, equality holds in the left inequality of (1.7) if and only if $K$ is centered, then if $K$ is not origin-symmetric, equality holds in the right inequality if and only if $\tau=0$.

For the case $n-p<i<n$ or $i>n$, the proof is similar.

The proof of Theorem 1.3 requires the following inequality (see [29]).

Lemma 3.2 If $f_{0}, f_{1}, \ldots, f_{m}$ are strictly positive continuous functions defined on $\mathcal{S}^{n-1}$ and $\lambda_{1}, \ldots, \lambda_{m}$ are positive constants the sum of whose reciprocals is unity, then

$$
\int_{\mathcal{S}^{n-1}} f_{0}(u) f_{1}(u) \cdots f_{m}(u) d u \leq \prod_{i=1}^{m}\left[\int_{\mathcal{S}^{n-1}} f_{0}(u) f_{i}^{\lambda_{i}}(u) d u\right]^{\frac{1}{\lambda_{i}}}
$$

with equality if and only if there exist positive constants $\alpha_{1}, \ldots, \alpha_{m}$ such that $\alpha_{1} f_{1}^{\lambda_{1}}(u)=\cdots=$ $\alpha_{m} f_{m}^{\lambda_{m}}(u)$ for all $u \in S^{n-1}$.

Proof of Theorem 1.3 For $K_{1}, \ldots, K_{n} \in S_{o}^{n}$, in (3.7), take $\lambda_{1}=\lambda_{2}=\cdots=\lambda_{m}$, and

$$
\begin{aligned}
& f_{0}=c_{p}^{(\tau)}\left(K_{1}, u\right) \cdots c_{p}^{(\tau)}\left(K_{n-m}, u\right) \quad\left(f_{0}=1 \text { if } m=n\right) \\
& f_{i}=c_{p}^{(\tau)}\left(K_{n-i+1}, u\right) \quad(1 \leq i \leq m)
\end{aligned}
$$


then it follows that

$$
\begin{aligned}
& \int_{\mathcal{S}^{n-1}} c_{p}^{(\tau)}\left(K_{1}, u\right) \cdots c_{p}^{(\tau)}\left(K_{n}, u\right) d u \\
& \quad \leq \prod_{i=1}^{m}\left[\int_{\mathcal{S}^{n-1}} c_{p}^{(\tau)}\left(K_{1}, u\right) \cdots c_{p}^{(\tau)}\left(K_{n-m}, u\right) c_{p}^{(\tau)}\left(K_{n-i+1}, u\right)^{m} d u\right]^{\frac{1}{m}} .
\end{aligned}
$$

By definition (1.1), this yields

$$
C_{p}^{(\tau)}\left(K_{1}, \ldots, K_{n}\right)^{m} \leq \prod_{i=1}^{m} C_{p}^{(\tau)}\left(K_{1}, \ldots, K_{n-m}, K_{n-i+1}, K_{n-i+1}, \ldots, K_{n-i+1}\right) .
$$

From the equality condition of inequality (3.7), we can see that equality holds in Theorem 1.3 if and only if $K_{n-m+1}, \ldots, K_{n}$ all have a similar general $L_{p}$-chord.

When $m=n$ in Theorem 1.3 and use (1.4), we have the following corollary.

Corollary 3.3 If $K_{1}, \ldots, K_{n} \in \mathcal{S}_{o}^{n}$ and $\tau \in[-1,1], p>0$, then

$$
C_{p}^{(\tau)}\left(K_{1}, \ldots, K_{n}\right)^{n} \leq C_{p}^{(\tau)}\left(K_{1}\right) C_{p}^{(\tau)}\left(K_{2}\right) \cdots C_{p}^{(\tau)}\left(K_{n}\right)
$$

with equality if and only if $K_{1}, \ldots, K_{n}$ all have a similar general $L_{p}$-chord.

Proof of Theorem 1.4 For $i<j<k$, by Hölder's inequality (see [32]), we have

$$
\begin{aligned}
& C_{p, i}^{(\tau)}(K, L)^{\frac{k-j}{k-i}} C_{p, k}^{(\tau)}(K, L)^{\frac{j-i}{k-i}} \\
& =\left(\frac{1}{n} \int_{\mathcal{S}^{n-1}} c_{p}^{(\tau)}(K, u)^{n-i} c_{p}^{(\tau)}(L, u)^{i} d u\right)^{\frac{k-j}{k-i}}\left(\frac{1}{n} \int_{\mathcal{S}^{n-1}} c_{p}^{(\tau)}(K, u)^{n-k} c_{p}^{(\tau)}(L, u)^{k} d u\right)^{\frac{j-i}{k-i}} \\
& \geq \frac{1}{n} \int_{\mathcal{S}^{n-1}} c_{p}^{(\tau)}(K, u)^{\frac{(n-i)(k-j)+(n-k)(j-i)}{k-i}} c_{p}^{(\tau)}(L, u)^{\frac{i(k-j)+k(j-i)}{k-i}} d u \\
& =\frac{1}{n} \int_{\mathcal{S}^{n-1}} c_{p}^{(\tau)}(K)^{n-j} c_{p}^{(\tau)}(L, u)^{j} d u \\
& =C_{p, j}^{(\tau)}(K, L),
\end{aligned}
$$

that is,

$$
C_{p, j}^{(\tau)}(K, L)^{k-i} \leq C_{p, i}^{(\tau)}(K, L)^{k-j} C_{p, k}^{(\tau)}(K, L)^{j-i}
$$

From the equality condition of Hölder's inequality, we know that equality holds in (1.10) if and only if $K$ and $L$ have a similar general $L_{p}$-chord.

Letting $i=0, k=n$ in Theorem 1.4, we have the following fact.

Corollary 3.4 If $K, L \in \mathcal{S}_{o}^{n}$ and $\tau \in[-1,1], p>0$, then for $0 \leq j \leq n$,

$$
C_{p, j}^{(\tau)}(K, L)^{n} \leq C_{p}^{(\tau)}(K)^{n-j} C_{p}^{(\tau)}(L)^{j}
$$


For $j<0$ or $j>n$, the inequality (3.8) is reversed, with equality if and only if $K$ and $L$ have a similar general $L_{p}$-chord.

Let $j=1$ in Corollary 3.4, we obtain the following dual Minkowski inequality for the general $L_{p}$-mixed chord integrals.

Corollary 3.5 If $K, L \in \mathcal{S}_{o}^{n}$ and $\tau \in[-1,1], p>0$, then

$$
C_{p, 1}^{(\tau)}(K, L)^{n} \leq C_{p}^{(\tau)}(K)^{n-1} C_{p}^{(\tau)}(L)
$$

with equality if and only if $K$ and $L$ have a similar general $L_{p}$-chord.

Now we prove the associated inequalities based on $L_{p}$-radial combinations.

Proof of Theorem 1.5 For $i \leq n-p$, it follows from Minkowski's inequality (see [32]) that

$$
\begin{aligned}
& C_{p, i}^{(\tau)}\left(\lambda \circ K \tilde{+}_{p} \mu \circ L\right)^{\frac{p}{n-i}} \\
&=\left[\frac{1}{n} \int_{\mathcal{S}^{n-1}} c_{p}^{(\tau)}\left(\lambda \circ K \tilde{+}_{p} \mu \circ L, u\right)^{n-i} d u\right]^{\frac{p}{n-i}} \\
&=\left[\frac{1}{n} \int_{\mathcal{S}^{n-1}}\left(f_{1}(\tau) \rho^{p}\left(\lambda \circ K \tilde{+}_{p} \mu \circ L, u\right)+f_{2}(\tau) \rho^{p}\left(\lambda \circ K \tilde{+}_{p} \mu \circ L,-u\right)\right)^{\frac{n-i}{p}} d u\right]^{\frac{p}{n-i}} \\
&=\left[\frac { 1 } { n } \int _ { \mathcal { S } ^ { n - 1 } } \left(\lambda f_{1}(\tau) \rho^{p}(K, u)+\lambda f_{2}(\tau) \rho^{p}(-K, u)\right.\right. \\
&\left.\left.+\mu f_{1}(\tau) \rho^{p}(L, u)+\mu f_{2}(\tau) \rho^{p}(-L, u)\right)^{\frac{n-i}{p}} d u\right]^{\frac{p}{n-i}} \\
& \leq {\left[\frac{1}{n} \int_{\mathcal{S}^{n-1}}\left(\lambda f_{1}(\tau) \rho^{p}(K, u)+\lambda f_{2}(\tau) \rho^{p}(-K, u)\right)^{\frac{n-i}{p}} d u\right]^{\frac{p}{n-i}} } \\
&+\left[\frac{1}{n} \int_{\mathcal{S}^{n-1}}\left(\mu f_{1}(\tau) \rho^{p}(L, u)+\mu f_{2}(\tau) \rho^{p}(-L, u)\right)^{\frac{n-i}{p}} d u\right]^{\frac{p}{n-i}} \\
&= \lambda C_{p, i}^{(\tau)}(K)^{\frac{1}{n-i}}+\mu C_{p, i}^{(\tau)}(L)^{\frac{1}{n-i}} .
\end{aligned}
$$

Similarly, we can prove the case of $n-p<i<n$ or $i>n$. From the equality conditions of Minkowski's inequality, we know that equality holds in Theorem 1.5 if and only if $K$ and $L$ have a similar general $L_{p}$-chord.

In order to prove Theorem 1.6, we require the following lemma which is an extended form of Beckenbach's inequality (see [33]) obtained by Dresher (see [34]) through the means of moment-space techniques.

Lemma 3.3 (The Beckenbach-Dresher inequality) If $p \geq 1 \geq r \geq 0, f, g \geq 0$, and $\phi$ is a distribution function, then

$$
\left(\frac{\int_{E}(f+g)^{p} d \phi}{\int_{E}(f+g)^{r} d \phi}\right)^{\frac{1}{p-r}} \leq\left(\frac{\int_{E} f^{p} d \phi}{\int_{E} f^{r} d \phi}\right)^{\frac{1}{p-r}}+\left(\frac{\int_{E} g^{p} d \phi}{\int_{E} g^{r} d \phi}\right)^{\frac{1}{p-r}},
$$

with equality if and only if the functions $f$ and $g$ are positively proportional. 
Moreover, the inverse Beckenbach-Dresher inequality was obtained by Li and Zhao (see [35]).

Lemma 3.4 (The Inverse Beckenbach-Dresher inequality) If $r \leq 0 \leq p \leq 1, f, g \geq 0$, and $\phi$ is a distribution function, then

$$
\left(\frac{\int_{E}(f+g)^{p} d \phi}{\int_{E}(f+g)^{r} d \phi}\right)^{\frac{1}{p-r}} \geq\left(\frac{\int_{E} f^{p} d \phi}{\int_{E} f^{r} d \phi}\right)^{\frac{1}{p-r}}+\left(\frac{\int_{E} g^{p} d \phi}{\int_{E} g^{r} d \phi}\right)^{\frac{1}{p-r}}
$$

with equality if and only if the functions $f$ and $g$ are positively proportional.

Proof of Theorem 1.6 By definitions (1.4) and (2.4), we have

$$
\begin{aligned}
& \left(\frac{C_{p, i}^{(\tau)}\left(K \tilde{+}_{p} L\right)}{C_{p, j}^{(\tau)}\left(K \tilde{+}_{p} L\right)}\right)^{\frac{p}{j-i}} \\
& =\left(\frac{\frac{1}{n} \int_{\mathcal{S}^{n-1}} c_{p}^{(\tau)}\left(K \tilde{+}_{p} L, u\right)^{n-i} d u}{\frac{1}{n} \int_{\mathcal{S}^{n-1}} c_{p}^{(\tau)}\left(K \tilde{+}_{p} L, u\right)^{n-j} d u}\right)^{\frac{p}{j-i}} \\
& =\left(\frac{\frac{1}{n} \int_{\mathcal{S}^{n-1}}\left[f_{1}(\tau) \rho^{p}\left(K \tilde{+}_{p} L, u\right)+f_{2}(\tau) \rho^{p}\left(K \tilde{+}_{p} L,-u\right)\right]^{\frac{n-i}{p}} d u}{\frac{1}{n} \int_{\mathcal{S}^{n-1}}\left[f_{1}(\tau) \rho^{p}\left(K \tilde{+}_{p} L, u\right)+f_{2}(\tau) \rho^{p}\left(K \tilde{+}_{p} L,-u\right)\right]^{\frac{n-j}{p}} d u}\right)^{\frac{p}{j-i}} \\
& =\left(\frac{\frac{1}{n} \int_{\mathcal{S}^{n-1}}\left[f_{1}(\tau) \rho^{p}(K, u)+f_{2}(\tau) \rho^{p}(-K, u)+f_{1}(\tau) \rho^{p}(L, u)+f_{2}(\tau) \rho^{p}(-L, u)\right]^{\frac{n-i}{p}} d u}{\frac{1}{n} \int_{\mathcal{S}^{n-1}}\left[f_{1}(\tau) \rho^{p}(K, u)+f_{2}(\tau) \rho^{p}(-K, u)+f_{1}(\tau) \rho^{p}(L, u)+f_{2}(\tau) \rho^{p}(-L, u)\right]^{\frac{n-j}{p}} d u}\right)^{\frac{p}{j-i}} \\
& \leq\left(\frac{\frac{1}{n} \int_{\mathcal{S}^{n-1}}\left[f_{1}(\tau) \rho^{p}(K, u)+f_{2}(\tau) \rho^{p}(-K, u)\right]^{\frac{n-i}{p}} d u}{\frac{1}{n} \int_{\mathcal{S}^{n-1}}\left[f_{1}(\tau) \rho^{p}(K, u)+f_{2}(\tau) \rho^{p}(-K, u)\right]^{\frac{n-j}{p}} d u}\right)^{\frac{p}{j-i}} \\
& +\left(\frac{\frac{1}{n} \int_{\mathcal{S}^{n-1}}\left[f_{1}(\tau) \rho^{p}(L, u)+f_{2}(\tau) \rho^{p}(-L, u)\right]^{\frac{n-i}{p}} d u}{\frac{1}{n} \int_{\mathcal{S}^{n-1}}\left[f_{1}(\tau) \rho^{p}(L, u)+f_{2}(\tau) \rho^{p}(-L, u)\right]^{\frac{n-j}{p}} d u}\right)^{\frac{p}{j-i}} \\
& =\left(\frac{C_{p, i}^{(\tau)}(K)}{C_{p, j}^{(\tau)}(K)}\right)^{\frac{p}{j-i}}+\left(\frac{C_{p, i}^{(\tau)}(L)}{C_{p, j}^{(\tau)}(L)}\right)^{\frac{p}{j-i}}
\end{aligned}
$$

inequality (1.13) is proved. Using the same method, we can see that when $j \geq n \geq i \geq n-p$, the reversed inequality of (1.13) is obtained.

From the equality condition of Lemma 3.3 and Lemma 3.4, we know that equality in inequality (1.13) if and only if $K$ and $L$ have a similar general $L_{p}$-chord.

Finally, we prove the interesting equality for the $L_{p}$-radial bodies.

Proof of Theorem 1.7 For $\forall \tau \in[-1,1]$,

$$
\begin{aligned}
C_{p, i}^{(\tau)}\left(\bar{\triangle}_{p} K\right) & =\frac{1}{n} \int_{\mathcal{S}^{n-1}} c_{p}^{(\tau)}\left(\bar{\triangle}_{p} K, u\right)^{n-i} d u \\
& =\frac{1}{n} \int_{\mathcal{S}^{n-1}}\left[f_{1}(\tau) \rho^{p}\left(\bar{\triangle}_{p} K, u\right)+f_{2}(\tau) \rho^{p}\left(\bar{\triangle}_{p}-K, u\right)\right]^{\frac{n-i}{p}} d u \\
& =\frac{1}{n} \int_{\mathcal{S}^{n-1}}\left[\frac{1}{2} f_{1}(\tau) \rho^{p}(K, u)+\frac{1}{2} f_{2}(\tau) \rho^{p}(-K, u)\right.
\end{aligned}
$$




$$
\begin{aligned}
& \left.+\frac{1}{2} f_{1}(\tau) \rho^{p}(-K, u)+\frac{1}{2} f_{2}(\tau) \rho^{p}(K, u)\right]^{\frac{n-i}{p}} d u \\
= & \frac{1}{n} \int_{\mathcal{S}^{n-1}}\left[\frac{1}{2} \rho^{p}(K, u)+\frac{1}{2} \rho^{p}(-K, u)\right]^{\frac{n-i}{p}} d u \\
= & \frac{1}{n} \int_{\mathcal{S}^{n-1}} c_{p}^{(0)}(K, u)^{n-i} d u \\
= & C_{p, i}(K),
\end{aligned}
$$

that is, $C_{p, i}^{(\tau)}\left(\bar{\triangle}_{p} K\right)=C_{p, i}(K)$.

\section{Competing interests}

The authors declare that they have no competing interests.

\section{Authors' contributions}

All authors contributed equally to the writing of this paper. All authors read and approved the final manuscript.

\section{Acknowledgements}

The authors are most grateful to the referees for the extraordinary attention they gave to our paper. Research is supported by the Natural Science Foundation of China (Grant No. 11371224).

\section{Received: 8 October 2015 Accepted: 2 February 2016 Published online: 12 February 2016}

\section{References}

1. Gardner, RJ: Geometric Tomography, 2nd edn. Cambridge University Press, Cambridge (2006)

2. Schneider, R: Convex Bodies: The Brunn-Minskowski Theory, 2nd edn. Cambridge University Press, Cambridge (2014)

3. Lu, FH: Mixed chord-integrals of star bodies. J. Korean Math. Soc. 47(2), 277-288 (2010)

4. Feng, YB, Wang, WD: General mixed chord-integrals of star bodies. Rocky Mt. J. Math. (2015, accepted)

5. Haberl, C, Schuster, FE: General $L_{p}$ affine isoperimetric inequalities. J. Differ. Geom. 83, 1-26 (2009)

6. Haberl, C: $L_{p}$-Intersection bodies. Adv. Math. 217, 2599-2624 (2008)

7. Haberl, C: Minkowski valuations intertwining with the special linear group. J. Eur. Math. Soc. 4(5), 1565-1597 (2012)

8. Haberl, C, Ludwig, M: A characterization of $L_{p}$ intersection bodies. Int. Math. Res. Not. 2006, Article ID 10548 (2006)

9. Haberl, C, Schuster, FE: Asymmetric affine $L_{p}$ Sobolev inequalities. J. Funct. Anal. 257(3), 641-658 (2009)

10. Ludwig, M: Minkowski valuations. Trans. Am. Math. Soc. 357, 4191-4213 (2005)

11. Ludwig, M: Intersection bodies and valuations. Am. J. Math. 128, 1409-1428 (2006)

12. Feng, YB, Wang, WD: General $L_{p}$-harmonic Blaschke bodies. Proc. Indian Acad. Sci. Math. Sci. 124(1), 109-119 (2014)

13. Feng, YB, Wang, WD, Lu, FH: Some inequalities on general $L_{p}$-centroid bodies. Math. Inequal. Appl. 18(1), 39-49 (2015)

14. Haberl, C, Schuster, FE, Xiao, J: An asymmetric affine Pólya-Szegö principle. Math. Ann. 352, 517-542 (2012)

15. Liu, CY, Ma, TY: p-Mixed chord-integrals of star bodies. Commun. Appl. Math. Comput. 27(2), 239-245 (2013)

16. Parapatits, L: SL(n)-Covariant $L_{p}$-Minkowski valuations. J. Lond. Math. Soc. 89, 397-414 (2014)

17. Parapatits, L: SL(n)-Contravariant $L_{p}$-Minkowski valuations. Trans. Am. Math. Soc. 366, 1195-1211 (2014)

18. Schuster, FE: Convolutions and multiplier transformations. Trans. Am. Math. Soc. 359, 5567-5591 (2007)

19. Schuster, FE: Crofton measures and Minkowski valuations. Duke Math. J. 154, 1-30 (2010)

20. Schuster, FE, Wannerer, T: GL(n) contravariant Minkowski valuations. Trans. Am. Math. Soc. 364, $815-826$ (2012)

21. Schuster, FE, Weberndorfer, M: Volume inequalities for asymmetric Wulff shapes. J. Differ. Geom. 92, $263-283$ (2012)

22. Wang, WD, Feng, YB: A general $L_{p}$-version of Petty's affine projection inequality. Taiwan. J. Math. 17(2), 517-528 (2013)

23. Wang, WD, Li, YN: Busemann-Petty problems for general $L_{p}$-intersection bodies. Acta Math. Sin. Engl. Ser. 31(5), 777-786 (2015)

24. Wang, WD, Li, YN: General $L_{p}$-intersection bodies. Taiwan. J. Math. (accepted)

25. Wang, WD, Ma, TY: Asymmetric $L_{p}$-difference bodies. Proc. Am. Math. Soc. 142, 2517-2527 (2014)

26. Wannerer, T: GL(n) equivariant Minkowski valuations. Indiana Univ. Math. J. 60, 1655-1672 (2011)

27. Weberndorfer, M: Shadow systems of asymmetric $L_{p}$ zonotopes. Adv. Math. 240, 613-635 (2013)

28. Yan, L, Wang, WD: The general $L_{p}$-mixed brightness integrals. J. Inequal. Appl. 2015, 190 (2015)

29. Lutwak, E: Dual mixed volumes. Pac. J. Math. 58(2), 531-538 (1975)

30. Lutwak, E: Extended affine surface area. Adv. Math. 85, 39-68 (1991)

31. Grinberg, E, Zhang, GY: Convolutions transforms and convex bodies. Proc. Lond. Math. Soc. 78(3), 77-115 (1999)

32. Hardy, GH, Littlewood, JE, Pólya, G: Inequalities. Cambridge University Press, Cambridge (1934)

33. Beckenbach, EF, Bellman, R: Inequalities, 2nd edn. Springer, Berlin (1965)

34. Dresher, M: Moment spaces and inequalities. Duke Math. J. 20, 261-271 (1953)

35. Li, XY, Zhao, CJ: On the $p$-mixed affine surface area. Math. Inequal. Appl. 17(2), 443-450 (2014) 